\title{
A Novel Robust Approach for Correspondence-Free Extrinsic Calibration
}

\author{
Hu, Xiao; Olesen, Daniel; Per, Knudsen
}

Published in:

2019 IEEE/RSJ International Conference on Intelligent Robots and Systems (IROS)

Link to article, DOI:

10.1109/iros40897.2019.8968447

Publication date:

2020

Document Version

Publisher's PDF, also known as Version of record

Link back to DTU Orbit

Citation (APA):

Hu, X., Olesen, D., \& Per, K. (2020). A Novel Robust Approach for Correspondence-Free Extrinsic Calibration. In 2019 IEEE/RSJ International Conference on Intelligent Robots and Systems (IROS) IEEE. https://doi.org/10.1109/iros40897.2019.8968447

\section{General rights}

Copyright and moral rights for the publications made accessible in the public portal are retained by the authors and/or other copyright owners and it is a condition of accessing publications that users recognise and abide by the legal requirements associated with these rights.

- Users may download and print one copy of any publication from the public portal for the purpose of private study or research.

- You may not further distribute the material or use it for any profit-making activity or commercial gain

- You may freely distribute the URL identifying the publication in the public portal

If you believe that this document breaches copyright please contact us providing details, and we will remove access to the work immediately and investigate your claim. 


\title{
A Novel Robust Approach for Correspondence-Free Extrinsic Calibration
}

\author{
Xiao $\mathrm{Hu}^{1}$, Daniel Olesen ${ }^{1}$, Knudsen Per $^{1}$
}

\begin{abstract}
Extrinsic calibration is a necessary step when using heterogeneous sensors for robotics applications. Most existing methods work under the assumption that the prior data correspondence is known. Considering data loss and false measurements, the correspondence may not be accessible in practice. To solve this problem without knowing the correspondence, several probabilistic methods have been proposed. However, an implicit restriction on input data limits their application. Therefore, in this paper, we propose a more stable correspondence-free method with two improvements that can relax the restrictions on inputs and improve the calibration accuracy. The first improvement finds consistent sets from raw inputs using screw invariants, which significantly improve the robustness in case of outliers and data loss. A new optimization method on matrix Lie group is proposed as the second improvement, which demonstrates better accuracy. The experimental results on both numerical and real data show the superiority and robustness of the proposed method.
\end{abstract}

\section{INTRODUCTION}

Extrinsic calibration for heterogeneous sensors is one of the most fundamental and important tasks for many robotics applications, e.g. sensor fusion, and Simultaneous Localization And Mapping (SLAM). In general, extrinsic calibration between two rigidly connected coordinate systems, as shown in Fig. 1, can be modeled as $\mathbf{A X}=\mathbf{X B}(\mathbf{A}, \mathbf{B}, \mathbf{X}$ are homogeneous matrices in $\mathbb{S E}(3)$ ), which also relates to the hand-eye calibration problem. The previous work [1] has proved that at least two motion pairs with nonparallel rotation axes are needed to estimate $\mathbf{X}$. If the data correspondence is known, this problem can be solved with certificated global optimality by approach [2]. However, finding pairwise correspondence could be challenging in practice in case of package loss during data transmission or outliers generated by abnormal sensor measurements. Therefore, several probabilistic methods have been proposed recently to solve the calibration problem without the need to know the correspondence. Although the pairwise correspondence is not essential for those methods, there is still an implicit assumption that the two sets should be bijective. In other words, every data in set $\{\mathbf{A}\}$ must have a corresponding match in set $\{\mathbf{B}\}$, and vice versa. If this assumption does not hold, then their performance will drop dramatically, which constrains the application of those methods in practice.

\footnotetext{
*This work was financially supported by the Innovation Fund Denmark through the project Unmanned Aerial Vehicle for high-Quality Magnetic Surveying (UAV-QMS).

${ }^{1}$ Xiao $\mathrm{Hu}$, Daniel Olesen, and Knudsen Per are with the $\mathrm{Na}-$ tional Space Institute, Technical University of Denmark, Elektrovej, 2800, Kgs. Lyngby, Denmark. danoleespace.dtu.dk, xiahaalspace.dtu.dk, pk@space.dtu.dk
}

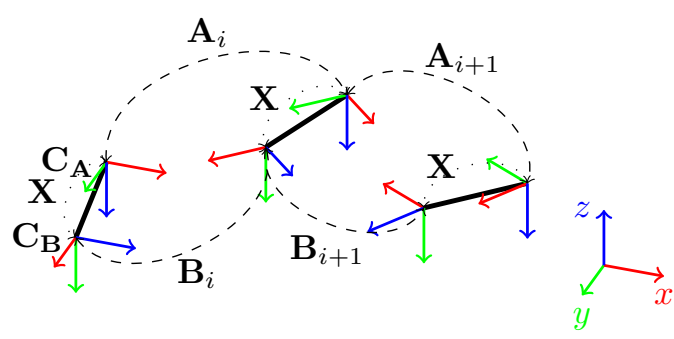

Fig. 1. Extrinsic calibration problem: $\mathbf{C}_{\mathbf{A}}, \mathbf{C}_{\mathbf{B}}$ represent the coordinate frames of sensor $A$ and sensor $B$, respectively. The corresponding transformation matrix from $\mathbf{C}_{\mathbf{B}}$ to $\mathbf{C}_{\mathbf{A}}$ is denoted with $\mathbf{X}$. The dashed connections are transformation matrices directly measured and dotted connections are transformation matrices to be estimated.

This paper focuses on solving the calibration problem without knowing the correspondence beforehand. By considering the restriction on data sets that required by the aforementioned correspondence-free approaches, we propose a method which can relax the restriction on data sets and improve the accuracy using a new optimization algorithm on matrix Lie group. The proposed method starts with a preprocessing modular to find consistent sets from the raw inputs by computing the consistency using screw invariants. After obtaining the consistent sets, we carry out the calibration using the proposed optimization approach. Compared with previous methods which use linearization, this optimization method works directly on special Euclidean group for better accuracy. Evaluations on synthetic data and real datasets have been carried out to validate the performance of the proposed method. The experimental results show the superiority and robustness of the proposed method against other correspondence-free approaches. When compared to state-of-the-art correspondence-dependent methods, it also shows stable performance. The main contributions of our work is that we propose a new algorithm using optimization on matrix Lie group which can improve estimation accuracy.

The remainder of the paper is organized as follows: Section II presents related work. Section III introduces the notation and mathematical preliminaries used in this paper. Section IV explains the details of the proposed approach. Section V describes the experiments and results. Finally, the conclusion is drawn in Section VI.

\section{RELATED WORK}

The hand-eye calibration problem was firstly studied in [3], followed by a considerable number of works on handeye calibration. Related work is reviewed in this section. 
The first category of methods attempts to solve the handeye calibration in a decoupled fashion, i.e. firstly the rotation estimation and followed by the estimation of the translational part. The majority of the earlier proposed approaches [1], [3]-[5] belong to this category. Their formulations vary with the parameterizations of the rotational part, e.g. axis and angle, unit quaternion, etc. Decouple rotation from translation yields efficient solutions, but at a cost that the rotation estimation errors would propagate to the translational part.

The second category of methods solves the hand-eye calibration jointly by estimating the rotation and translation simultaneously [4], [6]-[8]. The introduction of the screw theory in [9] provides a geometric view to analyze the restrictions of the hand-eye calibration problem, which later motivated the invention of the dual quaternion approach [6]. A similar linear formulation was proposed in [7] by using the Kronecker product. Due to noise, neither the constraint of the unit quaternion $(\|\mathbf{q}\|=1)$ nor the orthogonality constraint of $\mathbb{S O}(3)$ can be maintained, which requires normalization or orthogonalization. To suppress the influence of noise, iterative nonlinear optimization methods [4], [8] have been proposed. However, iterative approaches rely on good initial values for accuracy and fast convergence, while obtaining favorable initial values may be difficult in practice. Therefore, global optimization methods arise nowadays, which can perform calibration without the request for good initial values: e.g. using Second Order Cone Programming (SOCP) [10], using multivariate polynomial optimization [11], and by solving the Lagrange dual problem [2].

All the aforementioned approaches focus on solving the problem by assuming that the data correspondence is known in advance. To deal with motion pairs with unknown correspondences, several stochastic methods [12]-[14], which we summarize as the third category, have been proposed recently. The latest work by [13] proposed two novel batch methods that show better performance compared with approaches in [12], [14]. However, methods [12]-[14] share the common assumption that the two sets should be bijective. Put differently, every data in set $\{\mathbf{A}\}$ must have a corresponding match in set $\{\mathbf{B}\}$, and vice versa. In case that this assumption breaks, their performance will drop dramatically.

\section{NotATION \& PRELIMINARIES}

In the following sections, scalars are indicated with small letters (e.g. $f$ ). Vectors are indicated as small bold letters (e.g. r). Matrices are represented by bold capital letters (e.g. K).

\section{A. Matrix Lie Group}

The Lie algebra $\mathfrak{g}$ associated with an $n$-dimensional matrix Lie group is an $n$-dimensional tangent space. The mapping relationships are shown in Fig. 2. The exponential $\exp (\cdot)$ and logarithm $\log (\cdot)$ operations establish a local diffeomorphism between a neighborhood of $\mathbf{0}_{n \times n}$ in the tangent space to a local neighborhood of the identity on the manifold. $\mathfrak{g}$ associates to its vector space $\mathbb{R}^{n}$ by $(\cdot)^{\vee}: \mathfrak{g} \rightarrow \mathbb{R}^{n}$ and

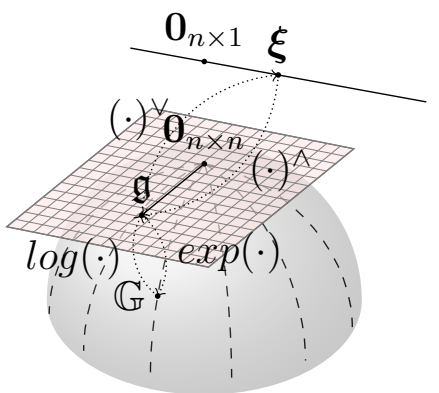

Fig. 2. Mapping relationship among matrix Lie group, Lie algebra, and the vector space.

$(\cdot)^{\wedge}: \mathbb{R}^{n} \rightarrow \mathfrak{g}$. The Lie algebra of $\mathfrak{s e}(3)$ is given as follows:

$$
\begin{aligned}
\boldsymbol{\xi}^{\wedge} & =\left[\begin{array}{l}
\boldsymbol{\rho} \\
\boldsymbol{\phi}
\end{array}\right]^{\wedge}=\left[\begin{array}{ll}
\boldsymbol{\phi}^{\wedge} & \boldsymbol{\rho} \\
\mathbf{0}^{T} & 0
\end{array}\right] \in \mathfrak{s e}(3) \\
\boldsymbol{\phi}^{\wedge} & =\left[\begin{array}{l}
\phi_{x} \\
\phi_{y} \\
\phi_{z}
\end{array}\right]^{\wedge}=\left[\begin{array}{ccc}
0 & -\phi_{z} & \phi_{y} \\
\phi_{z} & 0 & -\phi_{x} \\
-\phi_{y} & \phi_{x} & 0
\end{array}\right] \in \mathfrak{s o}(3)
\end{aligned}
$$

where $\phi, \rho \in \mathbb{R}^{3}$ and $\boldsymbol{\xi} \in \mathbb{R}^{6}$ being the coefficients in corresponding vector spaces. An additive increment in the vector space will associate to a multiplication increment on $\mathbb{S E}(3)$, which follows the following approximation [15]:

$$
\exp \left((\boldsymbol{\xi}+\delta \boldsymbol{\xi})^{\wedge}\right) \approx \exp \left(\left(\mathcal{J}_{l}^{-1} \delta \boldsymbol{\xi}\right)^{\wedge}\right) \exp \left(\boldsymbol{\xi}^{\wedge}\right)
$$

where $\mathcal{J}_{l}$ is the left Jacobian of $\mathbb{S E}(3)$ defined as (4):

$$
\mathcal{J}_{l}=\sum_{n=0}^{\infty} \frac{1}{(n+1)}\left(\boldsymbol{\xi}^{\wedge}\right)^{n}, \boldsymbol{\xi}^{\wedge}=\left[\begin{array}{l}
\boldsymbol{\rho} \\
\phi
\end{array}\right]^{\wedge}=\left[\begin{array}{ll}
\boldsymbol{\phi}^{\wedge} & \boldsymbol{\rho}^{\wedge} \\
\mathbf{0}^{T} & \boldsymbol{\phi}^{\wedge}
\end{array}\right]
$$

Finally, the adjoint $\mathcal{T}$ of $\mathbf{T} \in \mathbb{S E}(3)$ is defined as:

$$
\mathcal{T}=\left[\begin{array}{cc}
\mathbf{R} & \mathbf{t}^{\wedge} \mathbf{R} \\
\mathbf{0} & \mathbf{R}
\end{array}\right]
$$

\section{B. Probabilistic Solution Without Correspondence}

Probabilistic solutions for solving the calibration problem without knowing correspondence were proposed in [12][14]. Next, we briefly recall the necessary mathematical formulation. Assuming two data sets $\left\{\mathbf{A}: \mathbf{A}_{i}\right\},\{\mathbf{B}$ : $\left.\mathbf{B}_{i}\right\}, \mathbf{A}_{i}, \mathbf{B}_{i} \in \mathbb{S E}(3), i=1,2, \cdots, n$ and the pairwise correspondence is known, we have that $\mathbf{A}_{i} \mathbf{X}=\mathbf{X B}_{i}$. By using the probability theory on $\mathbb{S E}(3)$, the following two equations can be obtained as follows [13], which serves as the key for solving $\mathbf{X}$ without establishing correspondence.

$$
\begin{aligned}
\mathbf{M}_{\mathbf{A}} \mathbf{X} & =\mathbf{X M}_{\mathbf{B}} \\
\mathcal{T}_{\mathbf{X}^{-1}} \boldsymbol{\Sigma}_{\mathbf{A}} \mathcal{T}_{\mathbf{X}^{-1}}^{T} & =\boldsymbol{\Sigma}_{\mathbf{B}}
\end{aligned}
$$

where $\mathbf{M}_{(\cdot)}, \boldsymbol{\Sigma}_{(\cdot)}$ represent the mean and covariance, respectively. The discrete definition of the mean and covariance, taking $\mathbf{M}_{\mathbf{A}}$ and $\boldsymbol{\Sigma}_{\mathbf{A}}$ for example, are given as:

$$
\begin{aligned}
& \sum_{i=1}^{n} \log \left(\mathbf{M}_{\mathbf{A}}^{-1} \mathbf{A}\right)=0 \\
& \boldsymbol{\Sigma}_{\mathbf{A}}=\sum_{i=1}^{n} \boldsymbol{\epsilon} \boldsymbol{\epsilon}^{T}
\end{aligned}
$$




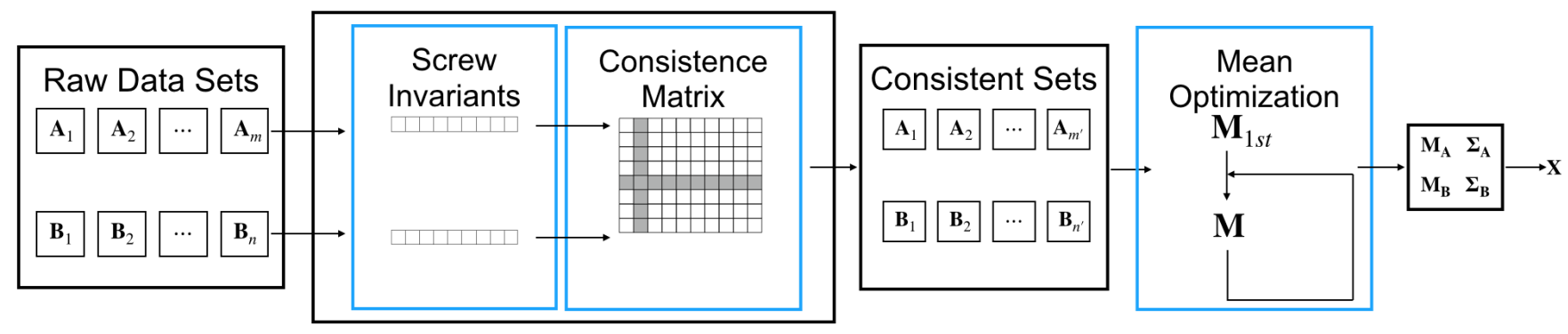

Fig. 3. Illustration of the proposed approach. It starts with finding consistent sets from raw inputs. The consistency is measured with the screw invariants. All pairwise combinations are checked to build a consistency matrix that is used for generating final consistent sets. Then consistent sets are fed to a new optimization method for estimating $\mathbf{M}_{\mathbf{A}}, \boldsymbol{\Sigma}_{\mathbf{A}}, \mathbf{M}_{\mathbf{B}}, \boldsymbol{\Sigma}_{\mathbf{B}}$. Finally, $\mathbf{X}$ is computed accordingly from $\mathbf{M}_{\mathbf{A}}, \boldsymbol{\Sigma}_{\mathbf{A}}, \mathbf{M}_{\mathbf{B}}, \boldsymbol{\Sigma}_{\mathbf{B}}$.

where $\boldsymbol{\epsilon}=\log \left(\mathbf{M}_{\mathbf{A}}^{-1} \mathbf{A}\right)^{\vee}$. According to [12], $\boldsymbol{\Sigma}$ can be further decomposed as: $\boldsymbol{\Sigma}=\left[\begin{array}{cc}\boldsymbol{\Sigma}^{1} & \left(\boldsymbol{\Sigma}^{2}\right)^{T} \\ \boldsymbol{\Sigma}^{2} & \boldsymbol{\Sigma}^{3}\end{array}\right]$. After some derivations, we will have:

$$
\begin{aligned}
\mathbf{R}_{\mathbf{X}}^{T} \boldsymbol{\Sigma}_{\mathbf{A}}^{3} \mathbf{R}_{\mathbf{X}} & =\boldsymbol{\Sigma}_{\mathbf{B}}^{3} \\
\mathbf{R}_{\mathbf{X}}^{T} \boldsymbol{\Sigma}_{\mathbf{A}}^{2} \mathbf{R}_{\mathbf{X}}\left(\left(-\mathbf{R}_{\mathbf{X}}^{T} \mathbf{t}_{\mathbf{X}}\right)^{\wedge}\right)^{T} & =\boldsymbol{\Sigma}_{\mathbf{B}}^{2}-\mathbf{R}_{\mathbf{X}}^{T} \boldsymbol{\Sigma}_{\mathbf{A}}^{2} \mathbf{R}_{\mathbf{X}}
\end{aligned}
$$

As described in [12], $\mathbf{R}_{\mathbf{X}}$ can be recovered from (6) and (10). Once $\mathbf{R}_{\mathbf{X}}$ is known, $\mathbf{t}_{\mathbf{X}}$ can be easily obtained from (11). To estimate $\mathbf{M}_{\mathbf{A}}$ and $\mathbf{M}_{\mathbf{B}}$, [13] proposed two batch methods that use linearization for $\mathbb{S E}(3)$. However, linearization will degrade accuracy. Hence, in Section IV, we propose a method using optimization on matrix Lie group to estimate the mean values for better accuracy.

\section{Proposed Method}

The proposed approach is depicted in Fig 3 and summarized in Algorithm 1.

\section{A. Finding Consistent Sets}

According to the Screw Congruence Theorem [9] and Euclidean group invariants [16], the rotation angle and the screw translation are invariants to coordinate transformations despite the motion being expressed in different coordinate systems. Therefore, these invariants are used as a measure to assess how likely a sample from one data set will have a correspondence in another data set. The computation of the rotation angle and screw translation $(\theta, d)$ from their corresponding $\mathbb{S E}(3)$ is as follows: apply the Rodrigues formula to obtain the rotation angle $\theta$ and rotation axis $\mathbf{r}$, then $d=\mathbf{t}^{T} \mathbf{r}$. Based on screw invariants $(\theta, d)$, we define the consistency $c$ of two motion $\mathbf{A}_{i}, \mathbf{B}_{j}$ as:

$$
c_{\mathbf{A}_{i}, \mathbf{B}_{j}}=w_{1}\left|\operatorname{angdiff}\left(\theta_{i}, \theta_{j}\right)\right|+w_{2}\left|d_{1}-d_{2}\right|
$$

where $\left(\theta_{i}, d_{i}\right),\left(\theta_{j}, d_{j}\right)$ are the screw invariants of $\mathbf{A}_{i}, \mathbf{B}_{j}$, $w_{1}$ and $w_{2}$ are weights with respect to rotation angle and translation, angdiff $(\cdot)$ computes the minimum distance of two angles. The consistency matrix is established by computing the consistency for all possible combinations of data pairs:

$$
\mathbf{C}=\left[\begin{array}{cccc}
c_{\mathbf{A}_{1}, \mathbf{B}_{1}} & c_{\mathbf{A}_{1}, \mathbf{B}_{2}} & \cdots & c_{\mathbf{A}_{1}, \mathbf{B}_{n}} \\
c_{\mathbf{A}_{2}, \mathbf{B}_{1}} & c_{\mathbf{A}_{2}, \mathbf{B}_{2}} & \cdots & c_{\mathbf{A}_{2}, \mathbf{B}_{n}} \\
\vdots & \vdots & \ddots & \vdots \\
c_{\mathbf{A}_{m}, \mathbf{B}_{1}} & c_{\mathbf{A}_{m}, \mathbf{B}_{2}} & \cdots & c_{\mathbf{A}_{m}, \mathbf{B}_{n}}
\end{array}\right]
$$

where the $i^{t h}$ row of $\mathbf{C}$ represents the consistencies of $\mathbf{A}_{i}$ relative to each element of set $\{\mathbf{B}\}$. Assuming $\mathbf{A}_{i}$ has a correspondence in set $\{\mathbf{B}\}$ in the noise-free condition, then the corresponding element in $i^{\text {th }}$ row of $\mathbf{C}$ must be zero. In practice, due to the noise, the corresponding consistency cannot guarantee to be zero, but within a threshold $\epsilon$, i.e. $\left|c_{\mathbf{A}_{i}, \mathbf{B}_{j}}\right|<\epsilon$. In contrast, if all consistencies of $\mathbf{A}_{i}$ to set $\{\mathbf{B}\}$ are over the threshold, then it is unlikely that $\mathbf{A}_{i}$ would have a correspondence in set $\{\mathbf{B}\}$. Thus, based on this point, we find the consistent set from set $\{\mathbf{A}\}$ by traversing each row of the consistency matrix $\mathbf{C}$ and collecting $\mathbf{A}_{i}$ if there is at least one consistency within the given threshold $\epsilon$. The consistent set for set $\{\mathbf{B}\}$ is found by the same operation to every column, which results in two consistent sets from the raw inputs as shown in Fig 3. It should be noted that this process does not aim to establish the pairwise correspondence by using screw invariants because screw invariants are relative measures which only serve as the necessary condition for the statement that two motion pairs are associated.

\section{B. Optimization of Mean on Matrix Lie Group}

Now we have two consistent sets from the raw input data sets, we start our probabilistic solution for recovering $\mathbf{X}$. Previous work [13] has shown a good approximation of mean can significantly improve the accuracy of $\mathbf{X}$. Here we propose a new iterative optimization algorithm to estimate mean. Unlike the second-order method proposed in [13] which uses linearization by relaxing the constraints imposed by $\mathbb{S O}(3)$, the proposed method directly optimizes on matrix Lie group, which will recover a more accurate $\mathbf{X}$, as shown in Sec V. Without loss of generality, the optimization algorithm is only illustrated on $\mathbf{M}_{\mathbf{A}}$ for brevity.

The nonlinear iterative optimization starts with an initial value which is computed with the first-order method [13]. Recalling the definition of mean in (8) and using the Taylor expansion of the matrix $\operatorname{logarithm}(\log (\mathbf{A})=$ $\left.\sum_{i=1}^{+\infty} \frac{(-1)^{i-1}}{i}(\mathbf{A}-\mathbf{I})^{i}\right)$, the first-order approximation of $\mathbf{M}$ should satisfy

$$
\frac{1}{n} \sum_{i=1}^{n}\left(\mathbf{M}_{\mathbf{A}}^{-1} \mathbf{A}_{i}-\mathbf{I}\right) \approx 0 \Leftrightarrow \mathbf{M}_{\mathbf{A}}^{-1}\left(\frac{1}{n} \sum_{i=1}^{n} \mathbf{A}_{i}\right)=\mathbf{I}
$$

Consequently, the first-order approximation of the mean is given as $\mathbf{M}_{\mathbf{A}}^{1 \text { st }}=\frac{1}{n} \sum_{i=1}^{n} \mathbf{A}_{i}$. Unfortunately, $\mathbf{M}_{\mathbf{A}}^{1 \text { st }}$ may not 
belong to $\mathbb{S E}(3)$ because the Euclidean mean of rotation matrices cannot guarantee to be within $\mathbb{S O}(3)$ [17]. To handle this problem, the singular value decomposition (SVD) is performed to find the orthogonal projection of the rotational part of $\mathbf{M}_{\mathbf{A}}^{1 s t}$ onto $\mathbb{S O}(3)$ [17] and then recompose $\mathbf{M}_{\mathbf{A}}^{1 s t}$ using the refined rotational part.

With $\mathbf{M}_{\mathbf{A}}^{1 s t}$ as the initial value, we then start the iterative optimization by optimizing the following objective function:

$$
f_{\text {opt }}=\frac{1}{n} \sum_{i=1}^{n}\left(\log \left(\mathbf{M}_{\mathbf{A}}^{-1} \mathbf{A}_{i}\right)\right)=0
$$

Considering the right small perturbation $\delta \boldsymbol{\xi} \in \mathbb{R}^{6}$ to current $\mathbf{M}_{\mathbf{A}}$ on its corresponding vector space, the perturbed transformation matrix $\tilde{\mathbf{M}}_{\mathbf{A}}$ is given as $\tilde{\mathbf{M}}_{\mathbf{A}}=\mathbf{M}_{\mathbf{A}} \exp \left(\delta \boldsymbol{\xi}^{\wedge}\right)$. The objective function after the perturbation is then given as:

$$
\begin{aligned}
f_{\text {opt }} & =\frac{1}{n} \sum_{i=1}^{n} \log \left(\tilde{\mathbf{M}} \mathbf{A} \mathbf{A}_{i}\right) \\
& =\frac{1}{n} \sum_{i=1}^{n} \log \left(\exp \left(-\delta \boldsymbol{\xi}^{\wedge}\right) \mathbf{M}_{\mathbf{A}}^{-1} \mathbf{A}_{i}\right)
\end{aligned}
$$

Since $\mathbf{M}_{\mathbf{A}}, \mathbf{A}_{i} \in \mathbb{S E}(3)$, with the closure property of matrix Lie Group, their product still belongs to $\mathbb{S E}(3)$. We use $\boldsymbol{\xi}_{i}^{\prime}$ to represent the Lie algebra of $\mathbf{M}_{\mathbf{A}}^{-1} \mathbf{A}_{i}$, i.e. $\mathbf{M}_{\mathbf{A}}^{-1} \mathbf{A}_{i}=$ $\exp \left(\boldsymbol{\xi}_{i}^{\prime \wedge}\right)$. Using (3), we will have the objective function as:

$$
f_{\text {opt }}=\frac{1}{n} \sum_{i=1}^{n} \log \left(\exp \left(-\mathcal{J}_{l}^{-1}\left(\boldsymbol{\xi}_{i}^{\prime}\right) \delta \boldsymbol{\xi}+\boldsymbol{\xi}_{i}^{\prime}\right)^{\wedge}\right)
$$

By taking the $(\cdot)^{\vee}$ operation and some algebraic operations, it can be further written in a normal equation as $\mathbf{A} \boldsymbol{x}=\boldsymbol{b}$ :

$$
\begin{gathered}
\frac{1}{n} \sum_{i=1}^{n}\left(-\mathcal{J}_{l}^{-1}\left(\boldsymbol{\xi}_{i}^{\prime}\right) \delta \boldsymbol{\xi}+\boldsymbol{\xi}_{i}^{\prime}\right)=0 \\
\Rightarrow \underbrace{\frac{1}{n} \sum_{i=1}^{n}\left(\mathcal{J}_{l}^{-1}\left(\boldsymbol{\xi}_{i}^{\prime}\right)\right)}_{\mathbf{A}} \underbrace{\delta \boldsymbol{\xi}}_{\boldsymbol{x}}=\underbrace{\frac{1}{n} \sum_{i=1}^{n}\left(\boldsymbol{\xi}_{i}^{\prime}\right)}_{\boldsymbol{b}}
\end{gathered}
$$

$\delta \boldsymbol{\xi}$ is solved with (18) and then used to update $\mathbf{M}_{\mathbf{A}}$ by $\mathbf{M}_{\mathbf{A}}=\mathbf{M}_{\mathbf{A}} \exp \left(\delta \boldsymbol{\xi}^{\wedge}\right)$. This optimization will be solved iteratively with the latest updated $\mathbf{M}_{\mathbf{A}}$ until a maximum iteration achieves or the norm of $\delta \boldsymbol{\xi}$ is smaller than a given threshold $\epsilon$. We finally summarize the proposed approach in Algorithm 1 for clarity.

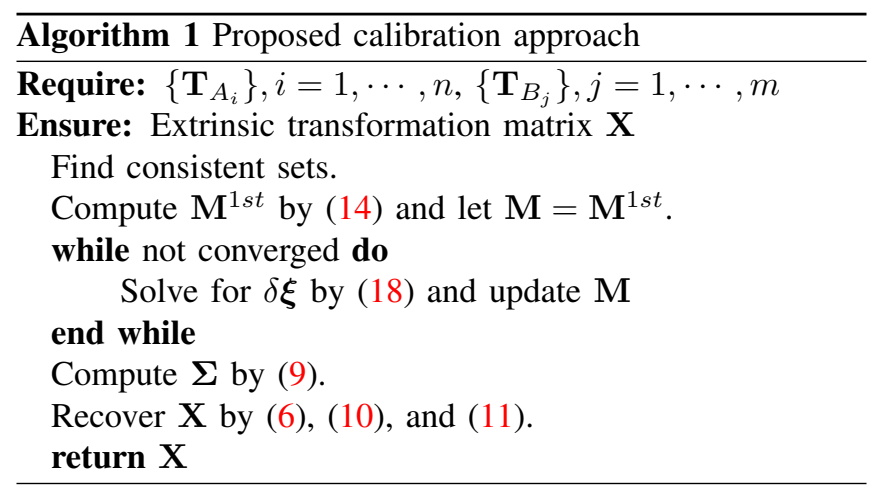

\section{EXPERIMENTS}

In this section, experiments on numerical data and real datasets are reported to validate the performance of the proposed method. In the first numerical experiment, we benchmark the performance of the proposed method with other state-of-the-art correspondence-free approaches. After that, the proposed method is compared with state-of-theart correspondence-dependent approaches on real datasets. Methods to be compared are listed as follows:

- the Batch1 (marked as B1) and Batch2 (B2) correspondence-free methods from [13] are used in the first experiment.

- the joint method (KR) [7], the iterative methods (NLQ by [4] and ATA) [8]), the SOCP optimization method (SOCP) from [10], the global optimization method using multivariate polynomial (GPOLY ${ }^{1}$ ) [11] and the DUAL method proposed in [2] are used in the second experiment.

Regarding the initial value for iterative methods, we use the default initialization routine of NLQ and ATA, see [4] and [8]. The proposed method is marked with BS throughout this section. All experiments are executed in MATLAB R2018A on a workstation with 2.80-GHz Intel Core i7 CPU and 32-GB RAM. The following error metrics are used to evaluate the results in both experiments:

$$
E_{\mathbf{R}}=\left\|\log \left(\hat{\mathbf{R}}_{X}^{T} \mathbf{R}_{\text {true }}\right)^{\vee}\right\|, \quad E_{\mathbf{t}}=\left\|\hat{\mathbf{t}}_{X}-\mathbf{t}_{\text {truth }}\right\|
$$

where $(\cdot)_{\text {truth }}$ represents the ground truth, $(\hat{\cdot})$ denotes the estimation, and $\|\cdot\|$ denotes the Euclidean norm.

\section{A. Numerical results of correspondence-free methods}

We divide the first experiment into three parts. The first part aims at evaluating the accuracy of the proposed mean optimization algorithm. The second and third parts are used to analyze the performance with respect to data loss and outliers.

To generate correspondence-free data, we firstly generate $\left\{\mathbf{A}_{i}, \mathbf{B}_{i}\right\}, i=1,2, \cdots, N$ with randomly selected ground truth $\mathbf{X}_{\text {truth }}$, i.e. $\mathbf{B}_{i}=\exp \left(\boldsymbol{\gamma}_{i}^{\wedge}\right) \exp \left(\boldsymbol{\zeta}_{i}^{\wedge}\right), \gamma_{i} \in$ $\mathcal{N}\left(\mathbf{0}, \sigma^{2} \mathbf{I}\right), \boldsymbol{\zeta}_{i} \in \mathcal{N}\left(\mathbf{0}, \sigma_{n}^{2} \mathbf{I}\right)$, then $\mathbf{A}_{i}=\mathbf{X}_{\text {truth }} \mathbf{B}_{i} \mathbf{X}_{\text {truth }}^{-1}$. Note $\boldsymbol{\zeta}_{i}$ represents the noise drawn from a zero-mean multivariate isometric Gaussian distribution with $\sigma_{n}$ denoting the standard deviation of the additional noise. Finally, $\left\{\mathbf{A}_{i}\right\},\left\{\mathbf{B}_{i}\right\}$ are completely scrambled. Moreover, a percentage $p_{\text {loss }}$ is used to simulate the situation of data loss. We compute the number of missing data as $N_{\text {loss }}=$ round $\left(p_{\text {loss }} * N\right)$ and then randomly discard $N_{\text {loss }}$ samples from one set. Similarly, regarding outliers, we generate $N_{\text {outlier }}=\operatorname{round}\left(p_{\text {outlier }} * N\right)$ outliers and place them randomly into one set. Consequently, the size of set $\{\mathbf{A}\}$ and set $\{\mathbf{B}\}$ may not necessarily be equal. Without loss of generality, we use $M$ and $N$ to represent the size of set $\{\mathbf{A}\}$ and set $\{\mathbf{B}\}$, respectively.

\footnotetext{
${ }^{1}$ The dual quaternion method "dqhec" is used for comparison since it is the best approach compared with another two formulations according to the results in [11].
} 

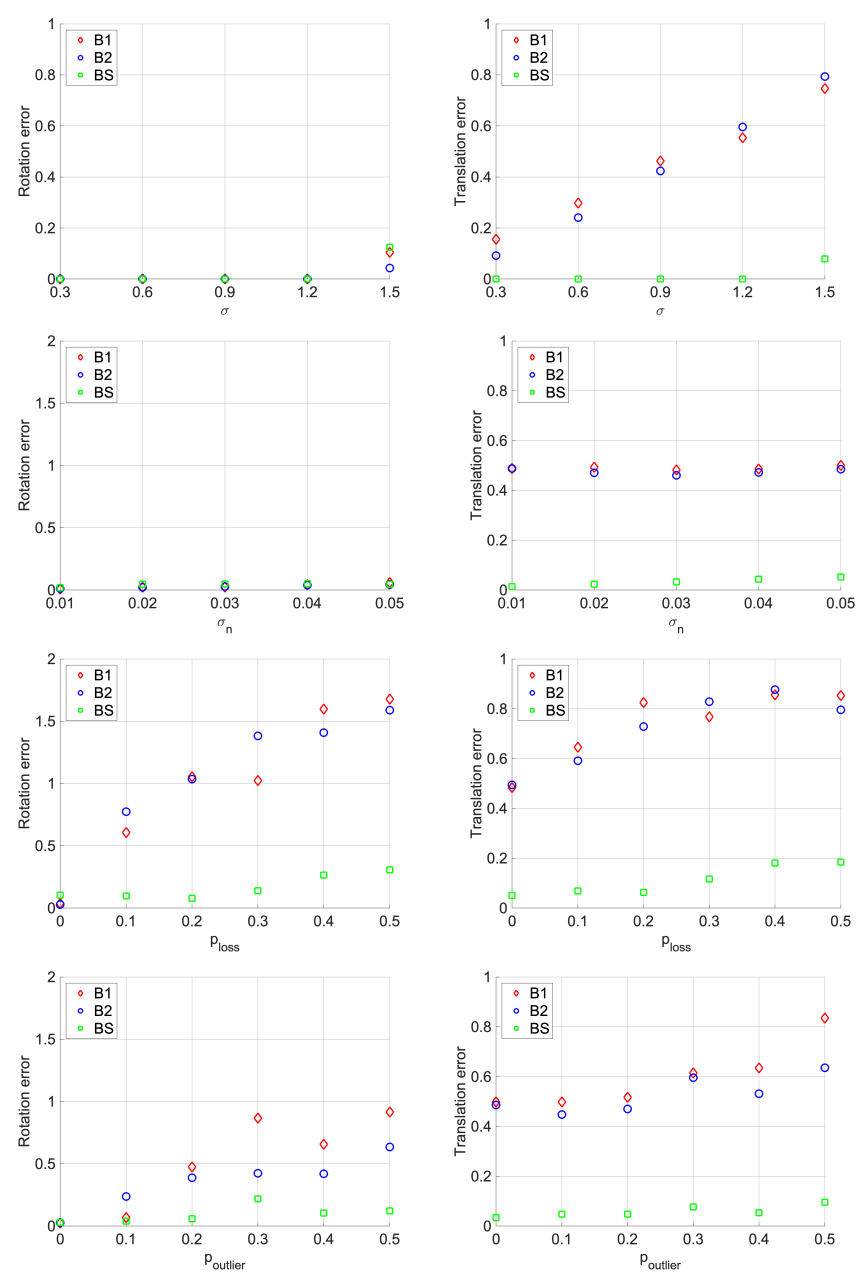

Fig. 4. Results of three cases simulated in the first experiment: the top two rows show the rotation and translation error plots for case 1 ( $M=N$, No Outliers), the third row shows the error plots for case 2 ( $M \neq N$, No Outliers), and the bottom row shows the error plots for case 3 ( $M \neq N$, Outliers). In all plots, the mean errors of 50 trials are taken as vertical ordinates.

1) $M=N$, No Outliers: We begin the first test by letting $M=N=50$ and including no outliers to compare the accuracy of the proposed mean optimization method with the accuracy of B1 and B2. By taking the mean values of 50 trials, we have the rotational and translational error plots shown in the top two rows of Fig 4. More specifically, the first top row shows the error plots by varying $\sigma(0.3-0.15$, stride $=0.3$ ) without exerting noise, while the second top row demonstrates the performance when we fix $\sigma=1$ and apply noise with the noise level $\sigma_{n}$ increasing from 0.01 to 0.05 by a step of 0.01 . Several observations can be made from these figures. Firstly, regarding rotation, all methods demonstrate approximately similar performance except BS behaves slightly worse than $\mathbf{B 1}$ and $\mathbf{B 2}$ when $\sigma$ goes to 1.5. Secondly, for translational accuracy, BS significantly outperforms B1 and B2 in all test cases.

2) $M \neq N$, No Outliers: In the second test, we aim to simulate the data loss situation which happens occasionally due to the reasons such as communication delay or package loss. Thus, $p_{\text {loss }}$ is varied from 0.0 to 0.5 with a step of 0.1 . Here, we set $\sigma=1$ and use a moderate noise level $\sigma_{n}=0.025$. Similarly to the previous test, 50 trials have been carried out and the mean errors for rotation and translation are used for analysis. The result is shown in the third row of Fig 4. As can be seen, only $\mathbf{B S}$ can obtain stable results regardless of the variation of $p_{\text {loss }}$. B1 and B2 demonstrate degenerate performance in this case with the errors diverging gradually. The result verifies the previous analysis that for batch methods in [13], although the pairwise correspondence is not a necessity, the two sets must be bijective. BS is capable of dealing with this case thanks to the proposed consistent set finding method.

3) $M \neq N$, Outliers: In the third test, we benchmark their performance against outliers. The same setting for $\sigma$ and $\sigma_{n}$ as the previous test is used here. Instead of varying $p_{\text {loss }}, p_{\text {outlier }}$ is increased from 0.0 to 0.5 with a stride of 0.1 . The mean errors for rotation and translation are shown in the bottom row of Fig 4. Once again, only BS can obtain accurate results, while the errors for B1 and $\mathbf{B 2}$ gradually diverge.

\section{B. Runtime Comparison}

The runtime for B1, B2, and BS is evaluated by varying the number of samples $(10-200$, stride $=10)$, which is shown in Fig 5. Firstly, BS shows a relatively longer runtime compared with B1 and B2. Secondly, all methods can converge within $600 \mathrm{~ms}$ when the number of samples is less than 200 .

Considering these observations, it can be concluded that 1) the proposed method shows superior performance in terms of estimating translation while maintaining a similar accuracy for estimating rotation; 2) the proposed method is capable of dealing with irregular cases including random outliers and data loss. However, the proposed method is relatively more time-consuming, which leaves as a future improvement.

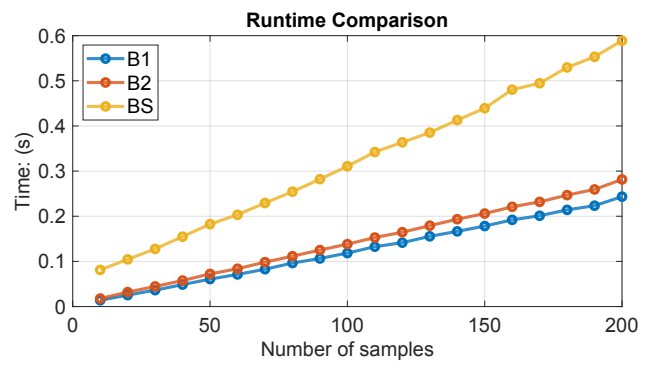

Fig. 5. Runtime comparison: the figure shows the evaluations of runtime versus the number of measurements. Mean runtime of 50 trials is used as the vertical ordinate.

\section{Real Datasets}

In the second experiment, we benchmark the performance of BS against correspondence-dependent methods on real datasets to evaluate its accuracy and robustness.

For a fair comparison, data correspondence is provided to correspondence-dependent methods (KR, NLQ, ATA, SOCP, GPOLY, DUAL). In the first comparison, the real RGB-D data provided by [18] is used. The results are 
TABLE I

QUANTITATIVE RESULT ON RGB-D DATASET

\begin{tabular}{c|c|c|c}
\hline Method & $E_{\mathbf{R}}:(\mathrm{rad})$ & $E_{\mathbf{t}}:(\mathrm{m})$ & Runtime: $(s)$ \\
\hline KR & 0.0310 & 0.0588 & 0.0221 \\
\hline NLQ & 0.0739 & 0.0233 & 1.1680 \\
\hline ATA & 0.0260 & 0.0127 & 0.5185 \\
\hline SOCP & 0.1005 & 0.1273 & 5.8258 \\
\hline GPOLY & 0.0255 & 0.0180 & 1.6093 \\
\hline DUAL & 0.0263 & 0.0125 & 0.9428 \\
\hline BS & 0.0280 & 0.0344 & 0.6671 \\
\hline \hline
\end{tabular}
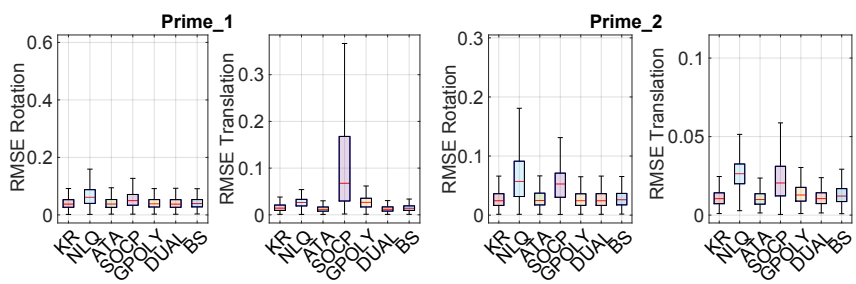

Fig. 6. Evaluations on RMSE of rotation and translation on datasets primesense_1 and primesense_2. For both datasets, the RMSE of rotation is shown in the left plot, whereas the RMSE of translation is shown in the right plot.

shown in Table. I. The rotational and translational errors are computed with respect to the ground truth provided in the dataset. Based on Table. I, GPOLY has the most accurate result for rotation estimation. Regard to translation, DUAL bypasses others. BS shows slightly worse accuracy compared with global optimization methods such as GPOLY, DUAL. In the second evaluation, the datasets primesense_l and primesense 2 from [19] are used. Since no ground truth is provided in those datasets, similarly to [19], the Root Mean Squared Error (RMSE) of the translation and orientation is used for evaluation. The boxplots in Fig. 6 represents the experimental results. For both datasets, we can see that except NLQ and SOCP, all other methods demonstrate stable calibration performance with slight differences.

To summarize from the second experiment, certain global optimization methods show their superiority in terms of accuracy. The proposed correspondence-free approach shows stable performance on real datasets, but the accuracy is slightly worse than some advanced global optimization methods. However, considering its capability of working under the correspondence-free condition, we can use the proposed method as the front-end for establishing data correspondence and find a good initial value, then refine the result using advanced global optimization methods for better accuracy.

\section{CONCLUSION}

In conclusion, this paper presents a new and stable correspondence-free extrinsic calibration approach. We apply a consistent set finding method that can detect and extract consistent sets from raw inputs, which significantly improves the robustness against outliers and missing data. A matrix Lie group based optimization algorithm is applied to estimate mean for better accuracy. Experiments have been carried out to evaluate the performance of the proposed method and other state-of-the-art approaches in terms of accuracy, stability, and runtime. The results show the superior performance of the proposed approach over the comparison approaches. Future work will be to speed up computation. Improve the performance against noise via leveraging softweighting scheme will also be considered.

\section{REFERENCES}

[1] R. Y. Tsai and R. K. Lenz, "A new technique for fully autonomous and efficient $3 \mathrm{~d}$ robotics hand/eye calibration," IEEE Transactions on robotics and automation, vol. 5, no. 3, pp. 345-358, 1989.

[2] M. Giamou, Z. Ma, V. Peretroukhin, and J. Kelly, "Certifiably globally optimal extrinsic calibration from per-sensor egomotion," IEEE Robotics and Automation Letters, 2018, submitted.

[3] Y. C. Shiu and S. Ahmad, "Calibration of wrist-mounted robotic sensors by solving homogeneous transform equations of the form ax= xb," IEEE Transactions on robotics and automation, vol. 5, no. 1, pp. 16-29, 1989.

[4] R. Horaud and F. Dornaika, "Hand-eye calibration," The international journal of robotics research, vol. 14, no. 3, pp. 195-210, 1995

[5] F. C. Park and B. J. Martin, "Robot sensor calibration: solving ax= $\mathrm{xb}$ on the euclidean group," IEEE Transactions on Robotics and Automation, vol. 10, no. 5, pp. 717-721, 1994.

[6] K. Daniilidis, "Hand-eye calibration using dual quaternions," The International Journal of Robotics Research, vol. 18, no. 3, pp. 286298, 1999.

[7] N. Andreff, R. Horaud, and B. Espiau, "On-line hand-eye calibration," in 3-D Digital Imaging and Modeling, 1999. Proceedings. Second International Conference on. IEEE, 1999, pp. 430-436.

[8] K. Pachtrachai, F. Vasconcelos, F. Chadebecq, M. Allan, S. Hailes, V. Pawar, and D. Stoyanov, "Adjoint transformation algorithm for hand-eye calibration with applications in robotic assisted surgery," Annals of biomedical engineering, vol. 46, no. 10, pp. 1606-1620, 2018.

[9] H. H. Chen, "A screw motion approach to uniqueness analysis of head-eye geometry," in Computer Vision and Pattern Recognition, 1991. Proceedings CVPR'91., IEEE Computer Society Conference on. IEEE, 1991, pp. 145-151.

[10] Z. Zhao, "Hand-eye calibration using convex optimization," in Robotics and Automation (ICRA), 2011 IEEE International Conference on. IEEE, 2011, pp. 2947-2952.

[11] J. Heller, D. Henrion, and T. Pajdla, "Hand-eye and robot-world calibration by global polynomial optimization," in Robotics and Automation (ICRA), 2014 IEEE International Conference on. IEEE, 2014, pp. 3157-3164.

[12] M. K. Ackerman, A. Cheng, and G. Chirikjian, "An informationtheoretic approach to the correspondence-free $\mathrm{ax}=\mathrm{xb}$ sensor calibration problem," in Robotics and Automation (ICRA), 2014 IEEE International Conference on. IEEE, 2014, pp. 4893-4899.

[13] Q. Ma, H. Li, and G. S. Chirikjian, "New probabilistic approaches to the $\mathrm{AX}=\mathrm{XB}$ hand-eye calibration without correspondence," in 2016 IEEE International Conference on Robotics and Automation, ICRA 2016, Stockholm, Sweden, May 16-21, 2016, 2016, pp. 4365-4371.

[14] H. Li, Q. Ma, T. Wang, and G. S. Chirikjian, "Simultaneous hand-eye and robot-world calibration by solving the $a x=y b$ problem without correspondence," IEEE Robotics and Automation Letters, vol. 1, no. 1, pp. 145-152, 2016.

[15] T. D. Barfoot and P. T. Furgale, "Associating uncertainty with threedimensional poses for use in estimation problems," IEEE Transactions on Robotics, vol. 30, no. 3, pp. 679-693, 2014.

[16] M. K. Ackerman, A. Cheng, B. Shiffman, E. Boctor, and G. Chirikjian, "Sensor calibration with unknown correspondence: Solving $\mathrm{ax}=\mathrm{xb}$ using euclidean-group invariants," in 2013 IEEE/RSJ International Conference on Intelligent Robots and Systems. IEEE, 2013, pp. 13081313.

[17] M. Moakher, "Means and averaging in the group of rotations," SIAM journal on matrix analysis and applications, vol. 24, no. 1, pp. 1-16, 2002

[18] J. Brookshire and S. Teller, "Extrinsic calibration from per-sensor egomotion," in Proceedings of Robotics: Science and Systems, Sydney, Australia, July 2012.

[19] F. Furrer, M. Fehr, T. Novkovic, H. Sommer, I. Gilitschenski, and R. Siegwart, "Evaluation of combined time-offset estimation and handeye calibration on robotic datasets," in Field and Service Robotics. Springer, 2018, pp. 145-159. 